Research Article

\title{
Combined Effect of NSI and SFP on Solar Electron Neutrino Oscillation
}

\author{
Deniz Yilmaz \\ Department of Physics Engineering, Faculty of Engineering, Ankara University, Tandogan, 06100 Ankara, Turkey \\ Correspondence should be addressed to Deniz Yilmaz; dyilmaz@eng.ankara.edu.tr
}

Received 12 January 2016; Revised 22 April 2016; Accepted 15 May 2016

Academic Editor: Theocharis Kosmas

Copyright (C) 2016 Deniz Yilmaz. This is an open access article distributed under the Creative Commons Attribution License, which permits unrestricted use, distribution, and reproduction in any medium, provided the original work is properly cited. The publication of this article was funded by $\mathrm{SCOAP}^{3}$.

\begin{abstract}
The combined effect of spin-flavor precession (SFP) and the nonstandard neutrino interaction (NSI) on the survival probability of solar electron neutrinos (assumed to be Dirac particles) is examined for various values of $\epsilon_{11}, \epsilon_{12}$, and $\mu B$. It is found that the neutrino survival probability curves affected by SFP and NSI effects individually for some values of the parameters $\left(\epsilon_{11}, \epsilon_{12}\right.$, and $\mu B$ ) get close to the standard MSW curve when both effects are combined. Therefore, the combined effect of SFP and NSI needs to be taken into account when the solar electron neutrino data obtained by low energy solar neutrino experiments is investigated.
\end{abstract}

\section{Introduction}

After first observation of the solar neutrino oscillation in Homestake neutrino experiment, serious solar, atmospheric, and reactor neutrino experiments were established to confirm it during the last decades. Both KamLAND experiment detecting reactor neutrinos $[1,2]$ and the combined analysis of the solar neutrino experiments (high precision water Cherenkov experiments SNO $[3,4]$ and SK $[5,6]$ and the radiochemical experiments Homestake [7], SAGE [8], GALLEX [9], and GNO [10]) strongly pointed out the socalled large mixing angle (LMA) region of the neutrino parameter space [11-16]. One of the implications of the physics beyond the Standard Model (SM) is the neutrino oscillation. Since neutrinos have a mass in a minimal extension of the SM, they have also magnetic moment [17]:

$$
\mu_{v}=\frac{3 e G_{f} m_{v}}{8 \pi^{2} \sqrt{2}}=\frac{3 e G_{f} m_{e} m_{v}}{4 \pi^{2} \sqrt{2}} \mu_{B}
$$

where $G_{f}$ is Fermi constant; $m_{e}$ and $m_{v}$ are the masses of electron and neutrino, respectively; and $\mu_{B}$ is Bohr magneton. While Majorana type neutrinos can only have off-diagonal (transition) magnetic moments, Dirac type neutrinos can have diagonal and off-diagonal magnetic moments $[18,19]$. If the neutrinos have magnetic moments, they can be effected by the large magnetic fields when they are passing through the magnetic region. Their spin can flip and the left-handed neutrino becomes a right-handed neutrino [20-24]. Thus the combined effect of the matter and the magnetic field called as spin-flavor precession (SFP) can change left-handed electron neutrino to another right-handed neutrino. This yields two other transitions $\left(v_{e_{L}} \rightarrow v_{\mu_{R}}\right.$ or $\left.\nu_{\tau_{R}}\right)$ in addition to the left-handed ones (i.e., in this scenario, the conversion probability is mainly affected) [24]. In the Dirac case, since the right-handed neutrinos are considered as sterile, they are not detectable by the detectors. On the other hand, if the neutrinos are of Majorana type, this conversion yields a solar antineutrino flux which is detectable by the detectors. These conversions for both Dirac and Majorana cases can also be responsible for the solar electron neutrino deficit. So far several studies related with the SFP have been studied in different aspects [25-31]. Astrophysical and cosmological arguments [32], Supernova 1987A [33, 34], solar neutrino experiments looking neutrino-electron scattering [35], and the reactor neutrino experiments $[36,37]$ provide some bounds on the neutrino magnetic moment. The new limit recently was obtained by GEMMA experiment: $\mu_{v}<2.9 \times 10^{-11} \mu_{B}$ at $90 \%$ CL [38]. However, another strong bound on neutrino transition magnetic moment was obtained in the presence of nonstandard neutrino-nucleus interactions by Papoulias 


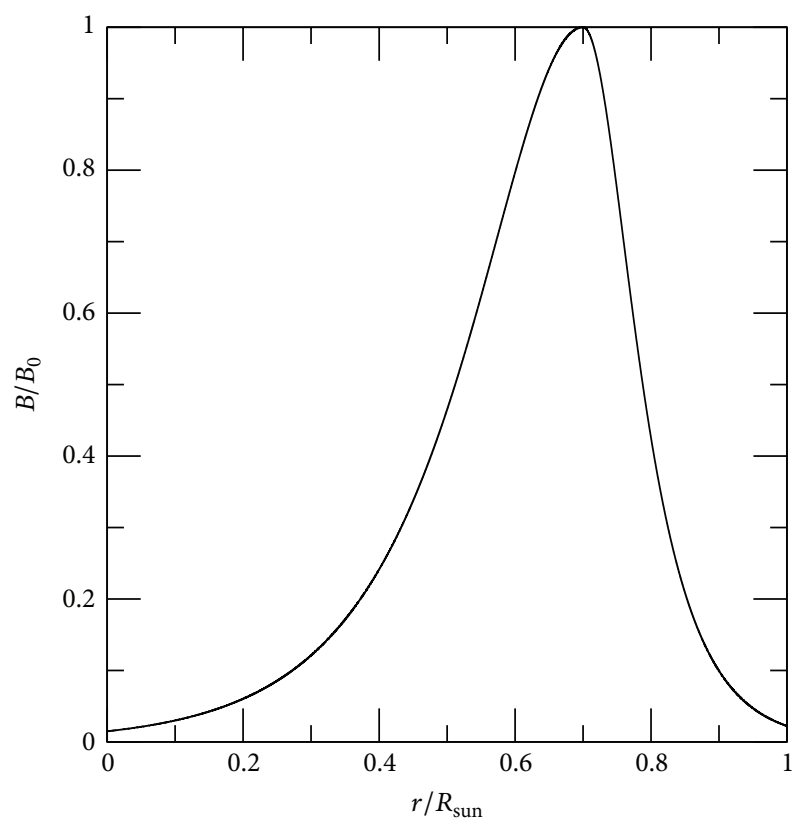

FIgURE 1: Magnetic field profile.

and Kosmas [39]. Detailed discussion on neutrino magnetic moment is also given elsewhere [40-45]. In addition to the knowledge about neutrino magnetic moment, the thorough information of solar magnetic fields is needed for the SFP analysis in the Sun. Despite the limited knowledge about it, some plausible profiles can be found in the literature [46, 47]. Standard solar model $[47,48]$ limits the solar magnetic field: 20 G near the solar surface [49], 20 kG-300 kG at the convective zone [47], and $<10^{7} \mathrm{G}$ at the solar center [47]. In this study the magnetic field profile is chosen as given in [46]. It has a peak at the bottom of the convective zone as shown in Figure 1.

Solar neutrinos can also be used for analyzing the physics beyond the Standard Model of the particle physics such as nonstandard forward scattering [50], mass varying neutrinos [51, 52], and long-range leptonic forces [53]. The probe of nonstandard neutrino interaction models is expected to observe in the transition region between $1 \mathrm{MeV}$ and $4 \mathrm{MeV}$ where the low energy solar neutrino experiments such as $\mathrm{SNO}+$ will examine. Even though the data is poor in this region, the studies comparing the effects of nonstandard models on the neutrino oscillation to the standard MSWLMA oscillation show that these effects modify the survival probability of neutrinos [50-56].

In this paper, the combined effect of nonstandard neutrino interactions (NSI) and SFP is examined in the case of two neutrino generations by assuming that the neutrinos are of Dirac type. The best fit LMA values are used for $\delta m_{12}^{2}$ and $\theta_{12}$ [57]. It is shown that the neutrino survival probability curves affected by SFP and NSI effects individually for some values of the parameters $\left(\epsilon_{11}, \epsilon_{12}\right.$, and $\left.\mu B\right)$ get close to the standard MSW curve when both effects are combined. Therefore, one can say that the combined effect of them needs to be taken into account when the solar electron neutrino data obtained by low energy solar neutrino experiments is investigated. Another analysis on the SFP effect in the presence of the NSI is examined for Majorana type solar neutrinos in [58].

\section{Spin-Flavor Precession (SFP) including Nonstandard Neutrino Interaction (NSI)}

The evolution equation including NSI matter effects in the SFP scenario for Dirac neutrinos can be written as

$$
i \frac{d}{d t}\left(\begin{array}{c}
v_{e_{L}} \\
v_{\mu_{L}} \\
v_{e_{R}} \\
v_{\mu_{R}}
\end{array}\right)=\left(\begin{array}{cc}
H_{L}+H_{\mathrm{NSI}} & B M^{\dagger} \\
B M & H_{R}
\end{array}\right)\left(\begin{array}{c}
v_{e_{L}} \\
v_{\mu_{L}} \\
v_{e_{R}} \\
v_{\mu_{R}}
\end{array}\right),
$$

where $H_{L}, H_{R}, H_{\mathrm{NSI}}$, and $M$ are the $2 \times 2$ submatrices and $B$ is the transverse magnetic field [24, 50]. For the Dirac neutrinos one writes down

$$
\begin{aligned}
& H_{L} \\
& =\left(\begin{array}{cc}
V_{c}+V_{n}+\frac{\delta m_{12}^{2}}{2 E} \sin ^{2} \theta_{12} & \frac{\delta m_{12}^{2}}{4 E} \sin 2 \theta_{12} \\
\frac{\delta m_{12}^{2}}{4 E} \sin 2 \theta_{12} & V_{n}+\frac{\delta m_{12}^{2}}{2 E} \cos ^{2} \theta_{12}
\end{array}\right),
\end{aligned}
$$

and $H_{R}=H_{L}\left(V_{c}=0=V_{n}\right)$. The matter potentials here are given as

$$
\begin{aligned}
& V_{c}=\sqrt{2} G_{F} N_{e}, \\
& V_{n}=-\frac{G_{F}}{\sqrt{2}} N_{n},
\end{aligned}
$$

where $N_{e}$ and $N_{n}$ are electron and neutron density, respectively [59-61]. The magnetic moment matrix for the Dirac neutrinos in (2) is written as [24]

$$
M=\left(\begin{array}{ll}
\mu_{e e} & \mu_{e \mu} \\
\mu_{\mu e} & \mu_{\mu \mu}
\end{array}\right) \text {. }
$$

The NSI contributions in (2) can be parametrized by fourfermion operator as given in [50]:

$$
=-2 \sqrt{2} G_{F}\left(\nu_{\alpha} \gamma_{\rho} v_{\beta}\right)\left(\epsilon_{\alpha \beta}^{f \bar{f} L} \bar{f}_{L} \gamma^{\rho} \bar{f}_{L}+\epsilon_{\alpha \beta}^{f \bar{f} R} \bar{f}_{R} \gamma^{\rho} \bar{f}_{R}\right),
$$$$
\mathscr{L}
$$

where $\epsilon^{f \bar{f} P}$ denotes the strength of the nonstandard interaction between $\alpha$ and $\beta$ types of neutrinos and the $P$ (left- or right-handed) components of the fermions $f$ and $\bar{f}$. Since the neutrino propagation can only be effected by the vector components where $f=\bar{f}$ of the nonstandard interaction $\left(\epsilon_{\alpha \beta}^{f}=\epsilon_{\alpha \beta}^{f f L}+\epsilon_{\alpha \beta}^{f f R}\right)$, one can define $\epsilon_{\alpha \beta}$ as the sum of the contributions from electrons, up quarks and down quarks in 

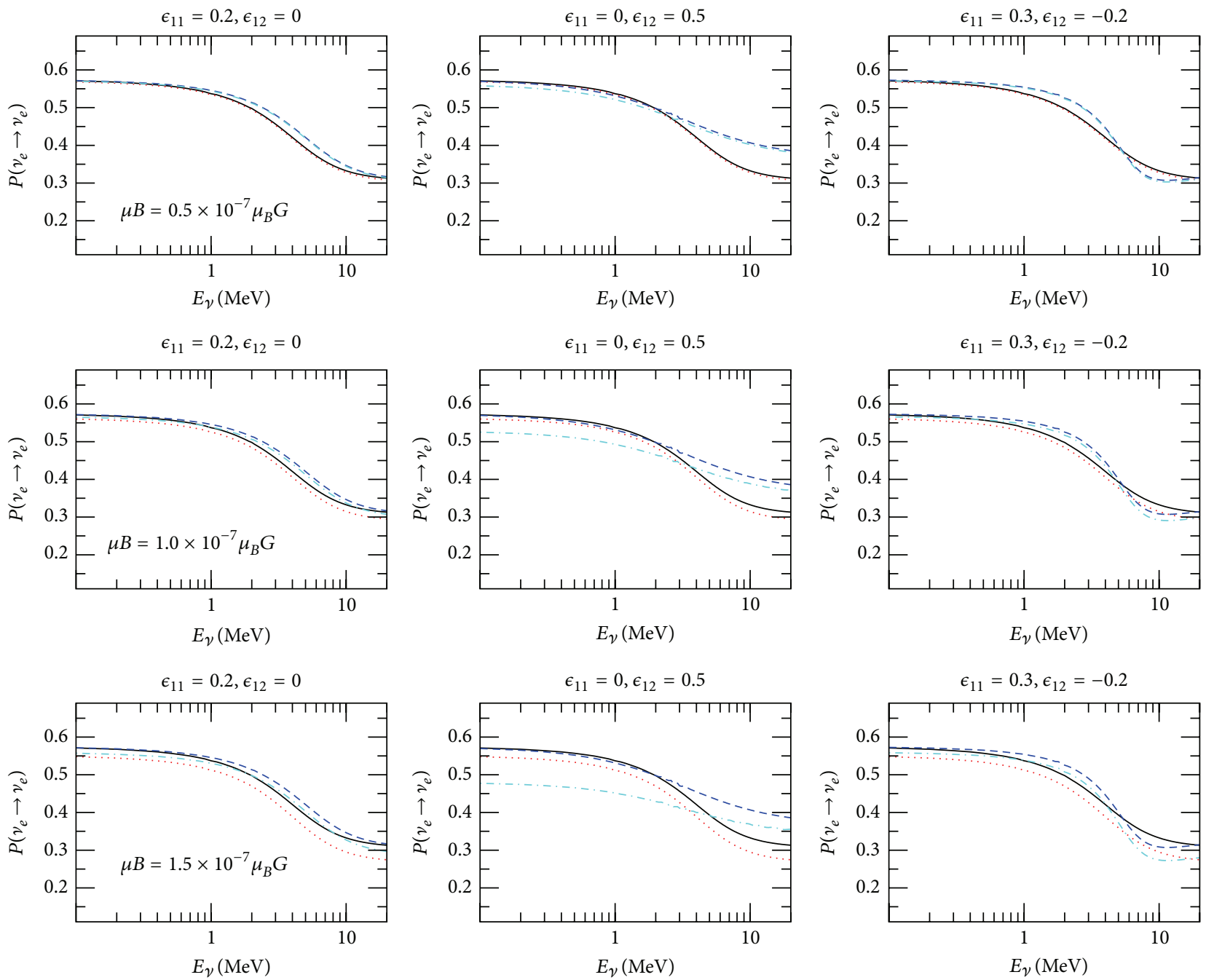

FIGURE 2: Survival probabilities for MSW-LMA prediction alone (solid lines), SFP effect at different $\mu B$ values (dotted lines), NSI effect alone (dashed lines), and the combined effect of the NSI and SFP (dotted-dashed lines). Each column uses the same $\epsilon_{11}$ and $\epsilon_{12}$ values, and each row uses the same $\mu B$ values.

matter: $\epsilon_{\alpha \beta}=\sum_{f=e, u, d} \epsilon_{\alpha \beta}^{f} N_{f} / N_{e}$. Then, the three-flavor NSI Hamiltonian can be written as

$$
H_{\mathrm{NSI}}^{3 \times 3}=V_{c}\left(\begin{array}{ccc}
\epsilon_{e e} & \epsilon_{e \mu}^{*} & \epsilon_{e \tau}^{*} \\
\epsilon_{e \mu} & \epsilon_{\mu \mu} & \epsilon_{\mu \tau}^{*} \\
\epsilon_{e \tau} & \epsilon_{\mu \tau} & \epsilon_{\tau \tau}
\end{array}\right) .
$$

After performing a rotation to $H_{\mathrm{NSI}}^{3 \times 3}$ by using the two factors of the neutrino mixing matrix, $T_{13} T_{23}$,

$$
T_{13}^{\dagger} T_{23}^{\dagger} H_{\mathrm{NSI}}^{3 \times 3} T_{13} T_{23},
$$

and decoupling the third flavor as in the standard threeflavor neutrino oscillation calculations, one can find the $2 \times 2$ neutrino nonstandard interaction (NSI) part in (2) as

$$
H_{\mathrm{NSI}}=V_{c}\left(\begin{array}{cc}
0 & \epsilon_{12}^{*} \\
\epsilon_{12} & \epsilon_{11}
\end{array}\right) \text {, }
$$

where $\epsilon_{11}$ and $\epsilon_{12}$ are the contributions from the new physics related to the original vectorial couplings, $\epsilon_{\alpha \beta}$, given as

$$
\begin{aligned}
\epsilon_{11} & =\epsilon_{\mu \mu} c_{23}^{2}-\left(\epsilon_{\mu \tau}+\epsilon_{\mu \tau}^{*}\right) s_{23} c_{23}+\epsilon_{\tau \tau} s_{23}^{2}-\epsilon_{e e} c_{13}^{2} \\
& +s_{13}\left[\left(e^{-i \delta} \epsilon_{e \mu}+e^{i \delta} \epsilon_{e \mu}^{*}\right) c_{13} s_{23}\right. \\
& \left.+\left(e^{-i \delta} \epsilon_{e \tau}+e^{i \delta} \epsilon_{e \tau}^{*}\right) c_{13} c_{23}\right]-s_{13}^{2}\left[\left(\epsilon_{\mu \tau}+\epsilon_{\mu \tau}^{*}\right) s_{23} c_{23}\right. \\
& \left.+\epsilon_{\mu \mu} s_{23}^{2}+\epsilon_{\tau \tau} c_{23}^{2}\right], \\
\epsilon_{12} & =c_{13}\left(\epsilon_{e \mu} c_{23}-\epsilon_{e \tau} s_{23}\right)+s_{13} e^{i \delta}\left[\epsilon_{\mu \tau} s_{23}^{2}-\epsilon_{\mu \tau}^{*} c_{23}^{2}\right. \\
& \left.-\left(\epsilon_{\mu \mu}-\epsilon_{\tau \tau}\right) s_{23} c_{23}\right] .
\end{aligned}
$$

Here $c_{i j}=\cos \theta_{i j}$ and $s_{i j}=\sin \theta_{i j}$ and $\delta$ is the CP-violating phase that we will ignore in our discussion [54].

The direct bounds on the NSI parameters come from atmospheric neutrino experiments (Super-Kamiokande, 

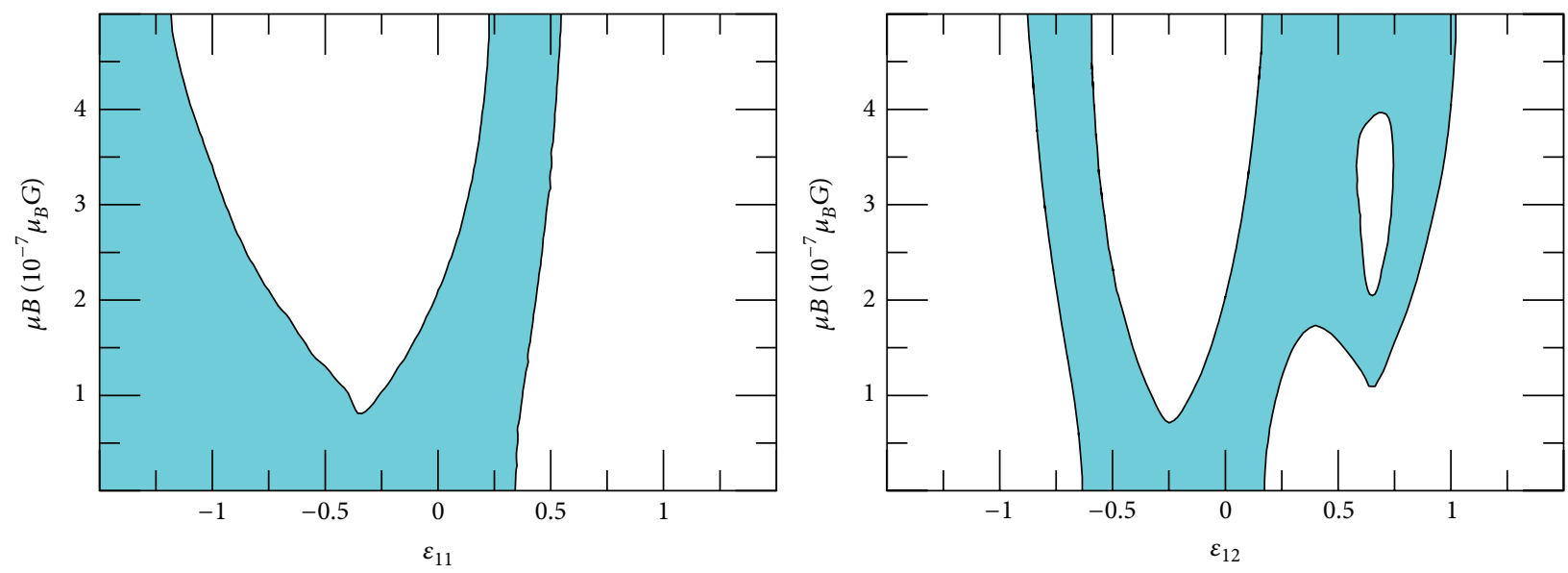

Figure 3: Allowed regions in the $\left(\epsilon_{11}, \mu B\right)$ and $\left(\epsilon_{12}, \mu B\right)$ planes at 90\% CL for $10 \mathrm{MeV}$ neutrino energy.

Ice-Cube-79) [62, 63], accelerator neutrino experiments (MINOS) [64], and some phenomenological studies [6568]: $\left|\epsilon_{e e}\right| \lesssim 0.5$ [62], $\left|\epsilon_{e \tau}\right| \lesssim 0.5$ [62], $\left|\epsilon_{\mu \tau}\right| \lesssim 6 \times 10^{-3}[63]$, $\left|\epsilon_{\tau \tau}-\epsilon_{\mu \mu}\right| \lesssim 3 \times 10^{-2}[63],-0.067 \leqslant \epsilon_{\mu \tau} \leqslant 0.023$ [64]. The effects of NSI were also studied by using data of reactor neutrino experiment, Daya Bay, [69] and solar neutrino experiments [70]. Detailed analysis on the nonstandard neutrino interactions and their limits is given in $[71,72]$.

\section{Results and Conclusions}

In this analysis the combined effect of the nonstandard neutrino interaction and SFP on the survival probability of solar electron neutrinos (assumed to be Dirac particles) is examined for various values of $\epsilon_{11}, \epsilon_{12}$, and $\mu B$. Results presented here are obtained numerically by diagonalizing the Hamiltonian in (2). In the calculations, the magnetic field profile given in Figure 1 is chosen as a Gaussian shape extending over the entire Sun [46] and the MSW-LMA best fit values are used: $\delta m_{12}^{2}=7.54 \times 10^{-5} \mathrm{eV}^{2}$ and $\sin ^{2} \theta_{12}=0.308$ [57].

Electron neutrino survival probabilities plotted as a function of neutrino energy are shown in Figure 2 for all situations: MSW-LMA prediction alone (solid lines), SFP alone (dotted lines), MSW-LMA + NSI (dashed lines), and SFP + NSI (dotted-dashed lines). In this figure, different from the SFP effect seen for all neutrino energies, the new physics effects change the standard MSW-LMA curve especially at the energies of $E \gtrsim 1 \mathrm{MeV}$ in which the region of $E \gtrsim 3.5 \mathrm{MeV}$ is well examined by the solar neutrino experiments $\mathrm{SNO}$ and SK. When the combined effect of them (SFP + NSI) is considered, the curves get closer to the standard curve than the curves affected by them individually for some values of the parameters $\left(\epsilon_{11}, \epsilon_{12}\right.$, and $\left.\mu B\right)$. A similar result was found in another analysis examined for Majorana neutrinos for only one NSI parameter, $\epsilon_{12}$ [58]. However, compared to the Dirac case presented here, SFP effect is seen at almost ten times larger $\mu B$ values in the Majorana case.

The allowed regions obtained by using the SNO results [73] are shown in Figure 3 in the $\left(\epsilon_{11}, \mu B\right)$ and $\left(\epsilon_{12}, \mu B\right)$ planes at $90 \% \mathrm{CL}$ for $10 \mathrm{MeV}$ neutrino energy. Even though the values of NSI parameters are expected to be very small $\left(\leqslant 10^{-2}\right)$, the large values of them are in the allowed regions when considering the SFP and NSI effects together. It is seen that the current solar neutrino data constrain the $\mu B$ and $\left(\epsilon_{11}\right.$, $\epsilon_{12}$ ) values poorly. A practical limit on them can be expected from the data obtained by the new low energy $(1 \mathrm{MeV} \lesssim$ $E \lesssim 4 \mathrm{MeV}$ ) solar neutrino experiments such as $\mathrm{SNO}+[74]$ probing the evidence of new physics effect. However, as it can be seen from the analysis presented here, the combined effect of SFP and NSI needs to be taken into account when the solar electron neutrino data obtained by new solar neutrino experiments is analyzed.

\section{Competing Interests}

The author declares that there is no conflict of interests regarding the publication of this paper.

\section{References}

[1] K. Eguchi, S. Enomoto, K. Furuno et al., "First results from KamLAND: evidence for reactor antineutrino disappearance," Physical Review Letters, vol. 90, Article ID 021802, 2003.

[2] T. Araki, K. Eguchi, S. Enomoto et al., "Measurement of neutrino oscillation with KamLAND: evidence of spectral distortion," Physical Review Letters, vol. 94, no. 8, Article ID 081801, 2005.

[3] Q. R. Ahmad, R. C. Allen, T. C. Andersen et al., "Measurement of the rate of $\nu_{e}+d \rightarrow p \rightarrow p+p+e^{-}$interactions produced by ${ }^{8} B$ solar neutrinos at the sudbury neutrino observatory," Physical Review Letters, vol. 87, no. 7, Article ID 071301, 2001.

[4] B. Aharmim, Q. R. Ahmad, S. N. Ahmed et al., "Determination of the $v_{e}$ and total ${ }^{8} \mathrm{~B}$ solar neutrino fluxes using the Sudbury Neutrino Observatory Phase I data set," Physical Review C, vol. 75, Article ID 045502, 2007.

[5] S. Fukuda, Y. Fukuda, M. Ishitsuka et al., "Solar ${ }^{8} B$ and hep neutrino measurements from 1258 days of super-kamiokande data," Physical Review Letters, vol. 86, no. 25, p. 5651, 2001. 
[6] S. Fukuda, Y. Fukuda, M. Ishitsuka et al., "Determination of solar neutrino oscillation parameters using 1496 days of SuperKamiokande-I data," Physics Letters B, vol. 539, no. 3-4, pp. 179187, 2002.

[7] B. T. Cleveland, T. Daily, R. Davis Jr. et al., "Measurement of the solar electron neutrino flux with the homestake chlorine detector," The Astrophysical Journal, vol. 496, no. 1, p. 505, 1998.

[8] J. N. Abdurashitov, T. J. Bowles, M. L. Cherry et al., "Measurement of the solar neutrino capture rate by sage and implications for neutrino oscillations in vacuum," Physical Review Letters, vol. 83, no. 23, p. 4686, 1999.

[9] W. Hampel, J. Handt, G. Heusser et al., "GALLEX solar neutrino observations: results for GALLEX IV," Physics Letters B, vol. 447, no. 1-2, pp. 127-133, 1999.

[10] M. Altmann, M. Balata, P. Belli et al., "GNO solar neutrino observations: results for GNO I," Physics Letters B, vol. 490, no. 1-2, pp. 16-26, 2000.

[11] A. B. Balantekin and H. Yuksel, "Global analysis of solar neutrino and KamLAND data," Journal of Physics G: Nuclear and Particle Physics, vol. 29, no. 4, p. 665, 2003.

[12] J. N. Bahcall and C. Pena-Garay, "A road map to solar neutrino fluxes, neutrino oscillation parameters, and tests for new physics," Journal of High Energy Physics, vol. 2003, no. 11, p. 4, 2003.

[13] M. C. Gonzalez-Garcia and C. Peña-Garay, "Three-neutrino mixing after the first results from K2K and KamLAND," Physical Review D, vol. 68, no. 9, Article ID 093003, 2003.

[14] P. C. de Holanda and A. Y. Smirnov, "Homestake result, sterile neutrinos, and low energy solar neutrino experiments," Physical Review D, vol. 69, no. 11, Article ID 113002, 2004.

[15] G. L. Fogli, E. Lisi, A. Marrone, and A. Palazzo, "Evidence for Mikheyev-Smirnov-Wolfenstein effects in solar neutrino flavor transitions," Physics Letters B, vol. 583, no. 1-2, pp. 149-156, 2004.

[16] J. N. Bahcall, M. C. Gonzalez-Garcia, and C. Pena-Garay, "Solar neutrinos before and after neutrino 2004," Journal of High Energy Physics, vol. 2004, no. 8, article 016, 2004.

[17] W. J. Marciano and A. I. Sanda, "Exotic decays of the muon and heavy leptons in gauge theories," Physics Letters B, vol. 67, no. 3, pp. 303-305, 1977.

[18] J. Schechter and J. W. F. Valle, "Majorana neutrinos and magnetic fields," Physical Review D, vol. 24, no. 7, p. 1883, 1981, Erratum: Physical Review D, vol. 25, pp. 283, 1982.

[19] R. E. Shrock, "Electromagnetic properties and decays of Dirac and Majorana neutrinos in a general class of gauge theories," Nuclear Physics B, vol. 206, no. 3, pp. 359-379, 1982.

[20] L. B. Okun, M. B. Voloshin, and M. I. Vysotsky, "Electromagnetic properties of neutrino and possible semiannual variation cycle of the solar neutrino flux," Soviet Journal of Nuclear Physics, vol. 44, p. 440, 1986, Yadernaya Fizika, vol. 44, pp. 677680, 1986.

[21] E. K. Akhmedov, "Resonant amplification of neutrino spin rotation in matter and the solar-neutrino problem," Physics Letters B, vol. 213, no. 1, pp. 64-68, 1988.

[22] E. K. Akhmedov and M. Y. Khlopov, "Resonant amplification of neutrino oscillations in longitudinal magnetic field," Modern Physics Letters A, vol. 3, no. 5, pp. 451-457, 1988.

[23] R. Barbieri and G. Fiorentini, "The solar neutrino puzzle and the $\mathbf{v}_{\mathbf{L}} \rightarrow \mathbf{v}_{\mathbf{R}}$ conversion hypothesis," Nuclear Physics B, vol. 304, pp. 909-920, 1988.

[24] C.-S. Lim and W. J. Marciano, "Resonant spin-flavor precession of solar and supernova neutrinos," Physical Review D, vol. 37, no. 6, pp. 1368-1373, 1988.
[25] A. B. Balantekin, P. J. Hatchell, and F. Loreti, "Matter-enhanced spin-flavor precession of solar neutrinos with transition magnetic moments," Physical Review D, vol. 41, no. 12, pp. 35833593, 1990

[26] A. A. Bykov, V. Y. Popov, T. I. Rashba, and V. B. Semikoz, "Resonant spin-flavorprecession solution to the solar neutrino problem and electron antineutrinos from the sun," http://arxiv.org/ abs/hep-ph/0002174.

[27] E. K. Akhmedov and J. Pulido, "Solar neutrino oscillations and bounds on neutrino magnetic moment and solar magnetic field," Physics Letters B, vol. 553, no. 1-2, pp. 7-17, 2003.

[28] B. C. Chauhan, J. Pulido, and E. Torrente-Lujan, "KamLAND, solar antineutrinos, and the solar magnetic field," Physical Review D, vol. 68, no. 3, Article ID 033015, 2003.

[29] A. B. Balantekin and C. Volpe, "Does the neutrino magnetic moment have an impact on solar neutrino physics?" Physical Review D, vol. 72, no. 3, Article ID 033008, 2005.

[30] D. Yilmaz and A. U. Yilmazer, "Global analysis of the data from solar neutrinos having transition magnetic moments together with KamLAND," Journal of Physics G: Nuclear and Particle Physics, vol. 31, no. 1, p. 57, 2005.

[31] D. Yilmaz and A. U. Yilmazer, "Global analysis of solar neutrinos (assumed to be Majorana particles) together with the new KamLAND data," Journal of Physics G, vol. 31, pp. 1123-1131, 2005.

[32] G. G. Raffelt, "New bound on neutrino dipole moments from globular-cluster stars," Physical Review Letters, vol. 64, no. 24, pp. 2856-2858, 1990.

[33] J. M. Lattimer and J. Cooperstein, "Limits on the neutrino magnetic moment from SN1987A," Physical Review Letters, vol. 61, no. 1, pp. 23-26, 1988.

[34] R. Barbieri and R. N. Mohapatra, "Limit on the magnetic moment of the neutrino from supernova 1987a observations," Physical Review Letters, vol. 61, no. 1, pp. 27-30, 1988.

[35] D. W. Liu, Y. Ashie, S. Fukuda et al., "Limits on the neutrino magnetic moment using 1496 days of super-kamiokande-i solar neutrino data," Physical Review Letters, vol. 93, no. 2, Article ID 021802, 2004.

[36] H. T. Wong, H. B. Li, S. T. Lin et al., "Search of neutrino magnetic moments with a high-purity germanium detector at the Kuo-Sheng nuclear power station," Physical Review D, vol. 75, no. 1, Article ID 012001, 2007.

[37] Z. Daraktchieva, C. Amslerb, M. Avenierc et al., "Final results on the neutrino magnetic moment from the MUNU experiment," Physics Letters B, vol. 615, no. 3-4, pp. 153-159, 2005.

[38] A. G. Beda, V. B. Brudanin, V. G. Egorov et al., "Gemma experiment: the results of neutrino magnetic moment search," Physics of Particles and Nuclei Letters, vol. 10, no. 2, pp. 139-143, 2013.

[39] D. K. Papoulias and T. S. Kosmas, "Neutrino transition magnetic moments within the non-standard neutrino-nucleus interactions," Physics Letters B, vol. 747, pp. 454-459, 2015.

[40] J. F. Beacom and P. Vogel, "Neutrino magnetic moments, flavor mixing, and the Super-Kamiokande solar data," Physical Review Letters, vol. 83, no. 25, pp. 5222-5225, 1999.

[41] N. F. Bell, V. Cirigliano, M. J. Ramsey-Musolf, P. Vogel, and M. B. Wise, "Magnetic moments of Dirac neutrinos," AIP Conference Proceedings, vol. 842, no. 1, pp. 874-876, 2006.

[42] M. Gorchtein, N. F. Bell, M. J. Ramsey-Musolf, P. Vogel, and P. Wang, "Model independent naturalness bounds on magnetic moments of Majorana neutrinos," AIP Conference Proceedings, vol. 903, no. 1, pp. 287-290, 2007. 
[43] A. B. Balantekin, "Neutrino magnetic moment," in Proceedings of the Origin of Matter and Evolution of Galaxies: International Symposium on Origin of Matter and Evolution of Galaxies 2005: New Horizon of Nuclear Astrophysics and Cosmology, vol. 847 of AIP Conference Proceedings, p. 128, Tokyo, Japan, November 2005.

[44] A. Studenikin, "Neutrino magnetic moment: a window to new physics," Nuclear Physics B-Proceedings Supplements, vol. 188, pp. 220-222, 2009.

[45] C. Broggini, C. Giunti, and A. Studenikin, "Electromagnetic properties of neutrinos," Advances in High Energy Physics, vol. 2012, Article ID 459526, 47 pages, 2012.

[46] C. R. Das, J. Pulido, and M. Picariello, "Light sterile neutrinos, spin flavor precession, and the solar neutrino experiments," Physical Review D, vol. 79, no. 7, Article ID 073010, 9 pages, 2009.

[47] S. Couvidat, S. Turck-Chièze, and A. G. Kosovichev, "Solar seismic models and the neutrino predictions," Astrophysical Journal, vol. 599, no. 2, pp. 1434-1448, 2003.

[48] J. N. Bahcall and M. H. Pinsonneault, "What do we (not) know theoretically about solar neutrino fluxes?" Physical Review Letters, vol. 92, no. 12, Article ID 121301, 4 pages, 2004.

[49] H. M. Antia, S. M. Chitre, and M. J. Thompson, "The Sun's acoustic asphericity and magnetic fields in the solar convection zone," Astronomy and Astrophysics, vol. 360, pp. 335-344, 2000.

[50] A. Friedland, C. Lunardini, and C. Peña-Garay, "Solar neutrinos as probes of neutrino-matter interactions," Physics Letters B, vol. 594, no. 3-4, pp. 347-354, 2004.

[51] M. Cirelli, M. C. Gonzalez-Garcia, and C. Peña-Garay, "Mass varying neutrinos in the Sun," Nuclear Physics B, vol. 719, no. 1-2, pp. 219-233, 2005.

[52] V. Barger, P. Huber, and D. Marfatia, "Solar mass-varying neutrino oscillations," Physical Review Letters, vol. 95, no. 21, Article ID 211802, 4 pages, 2005.

[53] M. C. Gonzalez-Garcia, P. C. de Holanda, E. Massó, and R. Zukanovich Funchal, "Probing long-range leptonic forces with solar and reactor neutrinos," Journal of Cosmology and Astroparticle Physics, vol. 2007, no. 1, article 005, 2007.

[54] R. Bonventre, A. Latorre, J. R. Klein, G. D. Orebi Gann, S. Seibert, and O. Wasalski, "Nonstandard models, solar neutrinos, and large $\theta_{13}$," Physical Review D, vol. 88, no. 5, Article ID 053010, 2013.

[55] A. Palazzo and D. Phys. Rev, Phys. Rev. D, vol. 83, Article ID 101701, 2011, arXiv:1101.3875.

[56] A. B. Balantekin and A. Malkus, "Solar neutrino matter effects redux," Physical Review D, vol. 85, no. 1, Article ID 013010, 9 pages, 2012.

[57] K. A. Olive, K. Agashe, C. Amsler et al., "Review of particle physics," Chinese Physics C, vol. 38, no. 9, Article ID 090001, 2014.

[58] D. Yilmaz, "SFP effect on Majorana type solar neutrinos in the presence of nonstandard neutrino interactions," Turkish Journal of Physics, vol. 39, pp. 309-313, 2015.

[59] L. Wolfenstein, "Neutrino oscillations in matter," Physical Review D, vol. 17, no. 9, pp. 2369-2374, 1978.

[60] S. P. Mikheev and A. Yu. Smirnov, "Resonance amplification of oscillations in matter and spectroscopy of solar neutrinos," Soviet Journal of Nuclear Physics, vol. 42, pp. 913-917, 1985, Translated from Yadernaya Fizika, vol. 42, pp. 1441-1448, 1985.

[61] S. P. Mikheyev and A. Yu. Smirnov, "Resonant amplification of $v$ oscillations in matter and solar-neutrino spectroscopy," $\mathrm{Il}$ Nuovo Cimento C, vol. 9, no. 1, pp. 17-26, 1986.
[62] G. Mitsuka, K. Abe, Y. Hayato et al., "Study of nonstandard neutrino interactions with atmospheric neutrino data in SuperKamiokande I and II," Physical Review D, vol. 84, no. 11, Article ID 113008, 2011.

[63] A. Groß and IceCube Collaboration, "Atmospheric neutrino oscillations in IceCube," Nuclear Physics B-Proceedings Supplements, vol. 237-238, pp. 272-274, 2013.

[64] P. Adamson, G. Barr, M. Bishai et al., "Search for flavorchanging non-standard neutrino interactions by MINOS," Physical Review D, vol. 88, no. 7, Article ID 072011, 6 pages, 2013.

[65] S. Davidson, C. Pena-Garay, N. Rius, and A. Santamaria, "Present and future bounds on non-standard neutrino interactions," Journal of High Energy Physics, vol. 2003, no. 3, p. 11, 2003.

[66] C. Biggio, M. Blennow, and E. Fernandez-Martinez, "Loop bounds on non-standard neutrino interactions," Journal of High Energy Physics, vol. 2009, no. 3, p. 139, 2009.

[67] D. K. Papoulias and T. S. Kosmas, "Nuclear aspects of neutral current non-standard $\nu$-nucleus reactions and the role of the exotic $\mu^{-} \rightarrow e^{-}$transitions experimental limits," Physics Letters $B$, vol. 728, pp. 482-488, 2014.

[68] R. Leitner, M. Malinsky, B. Roskovec, and H. Zhang, "Nonstandard antineutrino interactions at Daya Bay," Journal of High Energy Physics, vol. 2011, article 1, 2011.

[69] I. Girardi, D. Meloni, and S. T. Petcov, "The Daya Bay and T2K results on $\sin ^{2} 2 \theta_{13}$ and non-standard neutrino interactions," Nuclear Physics B, vol. 886, pp. 31-42, 2014.

[70] M. C. Gonzalez-Garcia and M. Maltoni, "Determination of matter potential from global analysis of neutrino oscillation data," Journal of High Energy Physics, vol. 2013, article 152, 2013.

[71] O. G. Miranda and H. Nunokawa, "Non standard neutrino interactions: current status and future prospects," New Journal of Physics, vol. 17, no. 9, Article ID 095002, 2015.

[72] T. Ohlsson, "Status of non-standard neutrino interactions," Reports on Progress in Physics, vol. 76, no. 4, Article ID 044201, 2013.

[73] B. Aharmim, S. N. Ahmed, A. E. Anthony et al., "Combined analysis of all three phases of solar neutrino data from the Sudbury Neutrino Observatory," Physical Review C, vol. 88, no. 2, Article ID 025501, 27 pages, 2013.

[74] C. Kraus and S. J. M. Peetersa, "The rich neutrino programme of the SNO+ experiment," Progress in Particle and Nuclear Physics, vol. 64, no. 2, pp. 273-277, 2010. 

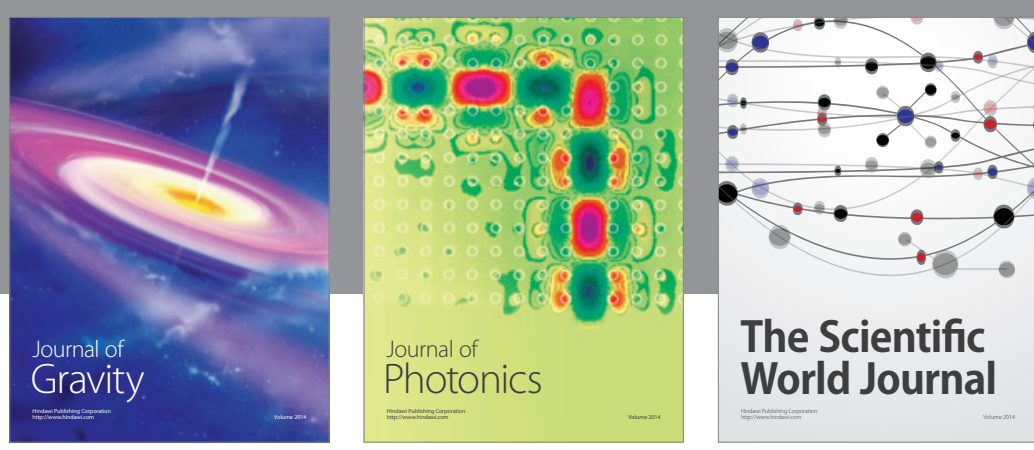

The Scientific World Journal
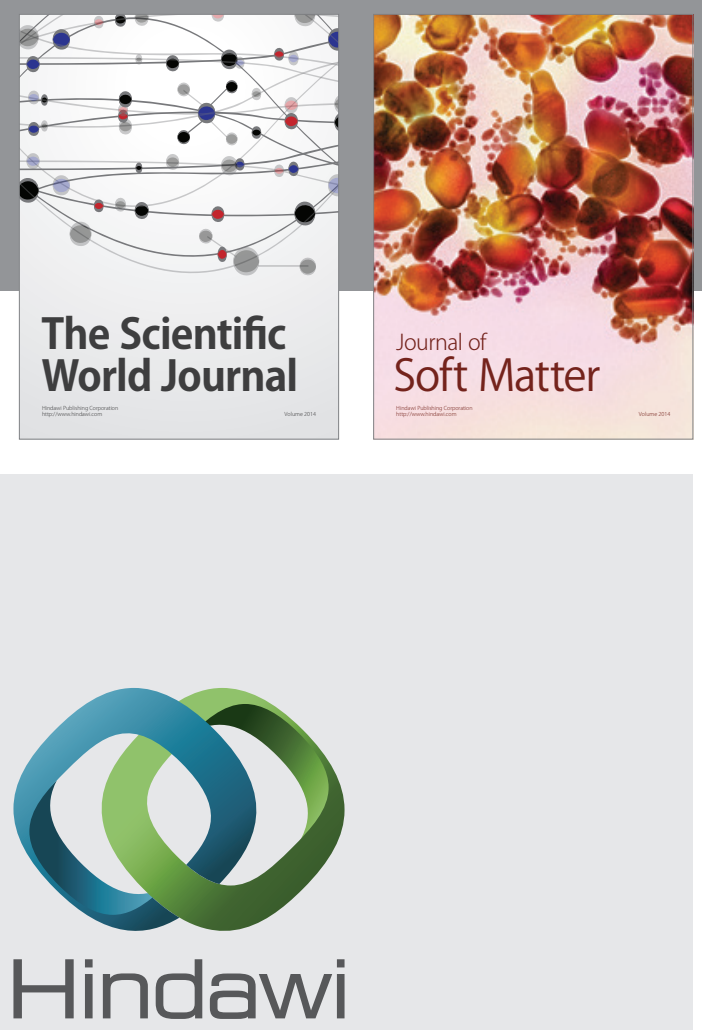

Submit your manuscripts at

http://www.hindawi.com

nternational Journal of

Statistical Mechanics
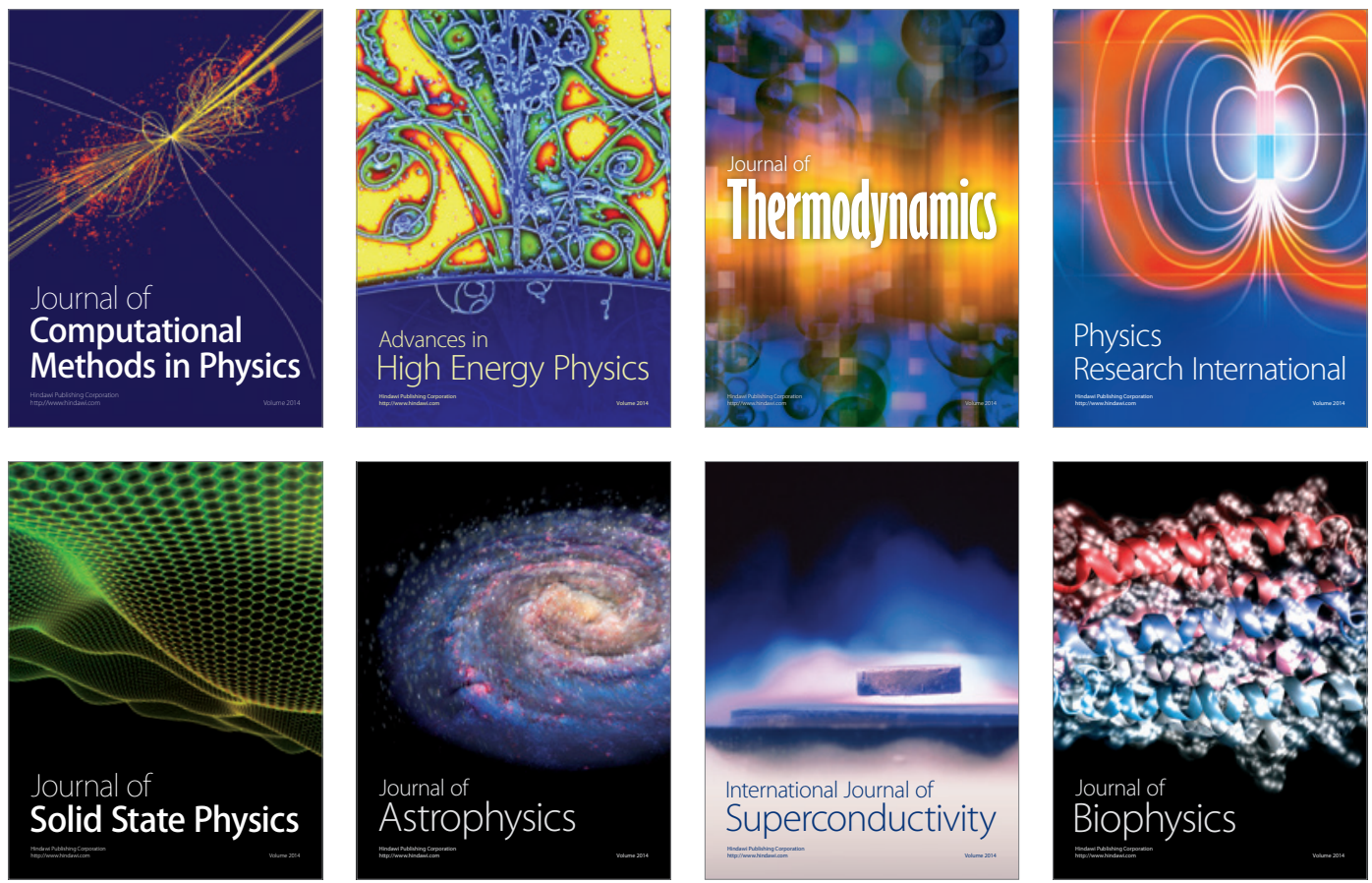
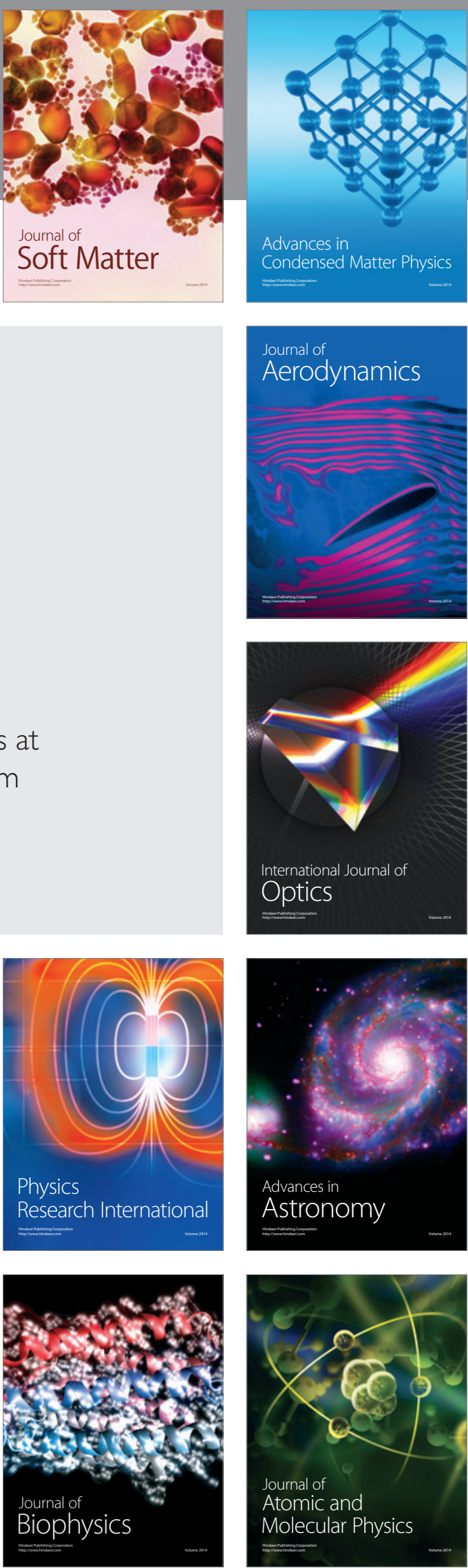